\title{
Reflexões sobre Cuidado e Aconselhamento de Profissionais de Saúde e Enfermeiros Em HIV/AIDS nos Centros de Testagem e Aconselhamento: Revisão Integrativa
}

\author{
Samara Maria de Jesus Veras ${ }^{1}$; Kerllane Rafaella Freire do Nascimento Santos ${ }^{1}$; \\ Claudia Daniele Barros Leite Salgueiro ${ }^{2}$; Silvana Cavalcanti dos Santos ${ }^{3}$; Cláudia Fabiane Gomes Gonçalves ${ }^{4}$; \\ Ana Carla Silva Alexandre ${ }^{5}$; Valdirene Pereira da Silva Carvalho ${ }^{6}$.
}

\begin{abstract}
Resumo: Objetivo: Evidenciar o perfil dos trabalhos publicados em âmbito nacional, no período de 2000 a 2017 sobre aconselhamento do Enfermeiro em HIV. Método: Revisão integrativa com o levantamento a partir do Portal da Biblioteca Virtual de Saúde, direcionando para periódicos indexados nas bases SciELO e LILACS. Resultados: Os resultados foram apresentados em uma tabela com a distribuição dos artigos e descrição de indicadores: ano de publicação, título, autor(es), nível de evidência científica, área de atuação dos pesquisadores e objetivos. Conclusão: Os autores encontraram ampla discussão na literatura, principalmente com enfoque na qualidade do aconselhamento prestados aos usuários, todavia, houve limitações quanto as publicações em relação a prática do cuidado em enfermagem as pessoas vivendo com HIV/AIDS atendidas no Centro de Testagem e Aconselhamento. O aconselhamento possui um papel importante no contexto da epidemia da AIDS no Brasil.
\end{abstract}

Palavras-chave: HIV; AIDS; aconselhamento; enfermagem; cuidado.

\section{Reflections about Care and Counseling of Health Professionals and Nurses in SIDA at the Test and Counseling Centers: Integrative Review}

Abstract: Objective: To demonstrate the profile of the works published at the national level, from 2000 to 2017. Method: Integrative review with the survey from the Portal of the Virtual Health Library, targeting journals indexed in the SciELO and LILACS databases. Results: The results were presented in a table with the distribution of articles and description of indicators: year of publication, title, author (s), level of scientific evidence, area of research and objectives. Conclusion: The authors found a wide discussion in the literature, mainly focusing on the quality of counseling provided to users, however, there were limitations regarding the publications regarding the practice of nursing care for people living with HIV / AIDS attended at the Testing and Counseling Center. Counseling plays an important role in the context of the AIDS epidemic in Brazil.

Keywords: HIV; AIDS; counseling; nursing; care.

\footnotetext{
${ }^{1}$ Discentes do curso de bacharelado em enfermagem - Instituto Federal de Educação, Ciência e Tecnologia de Pernambuco (IFPE) - Campus Pesqueira. ${ }^{2}$ Doutora em Psicologia Clínica pela Universidade Católica de Pernambuco (UNICAP), Mestra em Ciências da Saúde pela Universidade de Pernambuco (UPE), Pós Graduada em Saúde Coletiva pela mesma Universidade. Psicóloga formada pela Universidade Católica de Pernambuco (UNICAP). Docente do curso Bacharelado em Enfermagem do Instituto Federal de Educação, Ciência e Tecnologia de Pernambuco (IFPE) - Campus Pesqueira. Contato: claudia.leite@pesqueira.ifpe.edu.br.

${ }^{3}$ Mestra em Saúde Pública pelo Centro de Pesquisa Aggeu Magalhaes (CPqAM/FIOCRUZ), Pós Graduada em Saúde Pública pela Associação Caruaruense de Ensino Superior (ASCES). Enfermeira formada pela Universidade Estadual da Paraíba (UEPB). Docente do curso Bacharelado em Enfermagem do Instituto Federal de Educação, Ciência e Tecnologia de Pernambuco (IFPE) - Campus Pesqueira. Contato: silvana.santos@pesqueira.ifpe.edu.br.

${ }^{4}$ Mestra em Hebiatria pela pela Universidade de Pernambuco (UPE), Pós Graduada em Gestão dos Serviços e Sistemas de Saúde pelo Centro de Pesquisas Aggeu Magalhães (CPqAM). Enfermeira formada pela pela Universidade de Pernambuco (UPE). Docente do curso Bacharelado em Enfermagem do Instituto Federal de Educação, Ciência e Tecnologia de Pernambuco (IFPE) - Campus Pesqueira. Contato: claudia@ pesqueira.ifpe.edu.br.

${ }^{5}$ Doutora em Ciências da Saúde pelo Instituto Universitário Italiano de Rosário, Argentina (IUNIR), Pós Graduada em Unidade de Terapia Intensiva pelo Centro luniversitário Internacional (UNINTER) e em Emergência e Urgência (SES). Enfermeira formada pela Faculdade de Enfermagem de Arcoverde (FENFA). Docente do curso Bacharelado em Enfermagem do Instituto Federal de Educação, Ciência e Tecnologia de Pernambuco (IFPE) Campus Pesqueira. Contato: ana.alexandre@pesqueira.ifpe.edu.br .

${ }^{6}$ Pós Graduada em Enfermagem Obstétrica pela Universidade de Pernambuco (UPE), em Educação Profissional- Enfermagem pela Universidade Federal de Pernambuco (UFPE-FIOCRUZ) e em Saúde Pública pela Universidade de Ribeirão Preto (UNAERP). Enfermeira formada pela Universidade de Pernambuco (UPE). Docente do curso Bacharelado em Enfermagem do Instituto Federal de Educação, Ciência e Tecnologia de Pernambuco (IFPE) Campus Pesqueira. Contato: valdirene@pesqueira.ifpe.edu.br .
} 


\section{Introdução}

Com o advindo dos casos de Vírus da Imunodeficiência Humana (HIV) em 1980 e a identificação do vírus em 1984, a Sindrome da Imunodeficiencia Adquiriada (AIDS) se tranformou em um amplo problema de saúde pública. Problema este, que destaca-se entre as enfermidades infecciosas por sua magnitude e pela extenção dos danos causados às populações. Esta epidemia causada pelo HIV representa um fenômeno global, dinâmico e instável, cuja forma de ocorrência depende, dentre outros fatores, do comportamento humano convocando pesquisadores, gestores, profissionais de saúde a encontrar respostas efetivas para o seu enfrentamento deste processo de vida e morte que, embora com evolução crônica, requer mudanças de seus padrões de vida (TONNERA, 2015).

Com a inserção do Sistema Único de Saúde (SUS) na Constituição Federal Brasileira em 1988, a saúde passou a ser "direito de todos e dever do estado" em seu artigo 196 (BRASIL, 1988). Dentre os desafios que precisavam ser enfrentados na época, um deles era a epidemia da AIDS, que chegou durante esse período de transição democrática. Diante do contexto da década, ocorreram movimentos de luta contra essa doença que se instalava no país, preconizando por ações de promoção da saúde para as pessoas HIV positivas que necessitavam garantir seus direitos sociais "pautados por valores como dignidade, igualdade, respeito à diferença, tendo como principal estratégia o fortalecimento dos laços de solidariedade, resgate da alteridade, mobilização social e participação cívica"(BRASIL, 2011).

São notórios os impactos que o diagnóstico do HIV/aids, uma doença que não possui cura ainda, causando nos indivíduos, limitações físicas e psíquicas. Trazendo consigo medo do desconhecido, rejeição da sociedade, da sintomatologia e da morte. Mostrando também ansiedade, diminuição da autoestima, sensação de perda do controle, perda da função social e da expectativa de ter uma vida considerável comum (GARCIA; SOUZA, 2010).

De acordo com a UNAIDS (2015), todo o processo de cuidado e tratamento do HIV começa no dia em que uma pessoa é diagnosticada com infecção pelo vírus. O tratamento eficaz contra o HIV, não sugere apenas o uso de drogas, mas também abrange o acesso a serviços básicos e especializados que promovam a saúde e garantam a fixação do paciente ao longo do tratamento e consigam a eliminação duradoura da carga viral.

Ainda de acordo com o órgão não-governamental internacional, a rápida expansão do tratamento do HIV pode cooperar consideravelmente para o objetivo global de amortizar as 
desigualdades na saúde em todo o mundo. Apesar dos resultados obtidos até à data, medida em termos de aumento da esperança de vida, sejam genuínos, certas populações estão a se beneficiar muito menos do que outras (UNAIDS, 2015).

Em um contexto de desconhecimento e medo, com relação ao HIV/AIDS, foram criados no Brasil durante a década de 1980, Centros de Orientação e Aconselhamento Sorológico em DST/AIDS (COAS), posteriormente conhecido como CTA. Nestes serviços é possível realizar testes para detectar HIV, sífilis e hepatites B e C gratuitamente, além de disponibilizar o serviço de aconselhamento individual ou coletivo no pré e pós-teste aos usuários com o intuído de auxiliar no enfrentamento das IST/HIV/AIDS. (BRASIL, 2008; 2010). A equipe do CTA é formada por no mínimo um coordenador, profissionais responsáveis pela coleta, recepcionistas, apoio administrativos, segurança e aconselhadores (BRASIL, 2010).

O aconselhamento pode ser realizado por vários profissionais de saúde capacitados, dentre eles o enfermeiro. A prática do aconselhamento pode acontecer em qualquer âmbito em que os profissionais estejam inseridos, seja no âmbito da Estratégia de Saúde da família (PSF), nos ambulatórios, nos CTA ou até mesmo em hospitais (GRANEIRO et al, 2009).

Existe o aconselhamento individual, coletivo, para casais e continuado, para pessoas que aguardam os resultados dos exames, sendo esses, pré ou pós-teste. $\mathrm{O}$ aconselhamento pré-teste é uma parte do acolhimento, estabelecimento de vínculos, orientação sobre os testes e análise de vulnerabilidade, preservando o direito de o usuário querer ou não utilizar esse serviço. $\mathrm{O}$ pós-teste tem o objetivo de auxiliar adequadamente os usuários no enfrentamento do diagnóstico, bem como estimular práticas preventivas, independentemente dos resultados (BRASIL, 2010).

Os aconselhadores são responsáveis por uma prática complexa, onde deve haver respeito aos direitos humanos e à integralidade da atenção, tendo como base um discurso com proposta de mudança na atitude individual dos sujeitos para a redução de riscos, vulnerabilidades e agravos (BRASIL, 2010). Pode-se afirmar que o aconselhamento possui um papel importante no contexto da epidemia da AIDS no Brasil e tem se afirmado como um campo de conhecimento estratégico na qualidade do diagnóstico, assim como na atenção à saúde prestada aos usuários (PUPO, 2007; SOUZA et al, 2008).

O cuidar em enfermagem consiste em não medir esforços para auxiliar o outro, ajudando pessoas a encontrar forças mesmo na doença, sofrimento e dor, analisando todo o contexto de maneira holística. Em outras palavras, o cuidar é a maneira de demonstrar o saber fazer, o qual requer conhecimento e especialmente na atenção primáriaé uma competência do enfermeiro 
(SOUZA et al., 2008).

A enfermagem, visando a saúde mental dos indivíduos que apresentam diversas patologias, precisa enfatizar a promoção da saúde, a prevenção da enfermidade para ajudar o mesmo no enfrentamento e assistir ao paciente, à família e à comunidade em todas as necessidades (HIRDIS, 2000).

No perfil das modalidades que correspondem ao atendimento aos pacientes que vivem com HIV/AIDS, são contempladas ações curativas e preventivas. Ações estas que na ESF devem ser articuladas com os serviços de referência em HIV/aids de forma a intensificar o acompanhamento dos indivíduos que vivem com o vírus (SILVA et al. 2005).

Desse modo, se faz necessário que a Estratégia de Saúde da Família (ESF) trabalhe a promoção da saúde e a prevenção do HIV/Aids através de ações que permitam a orientação e o incentivo da relação sexual segura, tendo-se como necessário a utilização de preservativos durante as relações sexuais, assim como a disponibilização do acesso ao diagnóstico acompanhado pelo aconselhamento pré e pós teste anti-HIV (BRASIL, 2011).

Considera-se que é através do desgaste físico e psicológico que esses indivíduos sintam a necessidade e tenham expectativas voltadas para o aconselhamento e uma assistência mais individualizada, além de fazer com que percebam as diversas dimensões e o alcance de uma atenção à saúde com qualidade, podendo assim, contribuir no processo de restabelecimento da saúde (GOMES et al., 2012). Nesse contexto, este estudo teve por objetivo evidenciar o perfil dos trabalhos publicados em âmbito nacional, no período de 2000 a 2017 sobre aconselhamento do Enfermeiro em HIV.

\section{Metodologia}

Trata-se de uma revisão integrativa da literatura referente à produção do conhecimento sobre o cuidado e o aconselhamento do enfermeiro em HIV/Aids realizadas em CTA.

A revisão integrativa da literatura é considerada um instrumento essencial da prática baseada em evidências (PBE), tem o potencial de construir conhecimento fundamentado e uniforme para a realização de uma prática clínica de qualidade. Com o objetivo de proporcionar uma síntese dos conhecimentos e melhor aplicabilidade dos mesmos visando a compreensão da temática, a partir de outros estudos independentes (MENDES, 2008; BARROS, 2007). 
Esse método propõe o estabelecimento de critérios bem definidos sobre a coleta de dados, análise e apresentação dos resultados, desde o início do estudo, a partir de um protocolo de pesquisa previamente elaborado e validado. Para o desenvolvimento da presente revisão integrativa, foram percorridas seis etapas sugeridas por Souza, Silva e Carvalho (2009), a saber:

1. Identificação do tema ou formulação da questão norteadora: Para o presente estudo, formulou-se a seguinte questão norteadora: "Como é realizado o aconselhamento e o cuidado em HIV/AIDS por enfermeiros nos CTAs?"

Para referenciar as publicações que compuseram a revisão integrativa desse estudo, realizou-se uma busca online, nos meses de março e abril de 2017, com a classificação a partir do Portal Regional da Biblioteca Virtual de Saúde (BVS), com direcionamento à Scientific Electronic Library Online - SciELO e à Base de Dados da Literatura Latino-Americana e do Caribe em Ciências da Saúde - LILACS. Para tanto, foram empregados os seguintes descritores em Ciências da Saúde (DeCS):“HIV”, “AIDS”, "Enfermagem" e “Cuidado".

Optou-se por tais bases devido ao quantitativo de indexação de artigos da área da saúde e nas temáticas relacionadas à enfermagem. Os descritores foram combinados de diferentes formas, com o objetivo de ampliar a busca pelos estudos, foram consideradas as variações terminológicas e os sinônimos. Para a concretização de uma busca sensibilizada, procedeu-se com o uso do operador booleano "AND" para o evento simultâneo de assuntos.

2. Estabelecimento de critérios para inclusão e exclusão de estudos e realização da amostragem ou busca na literatura: Foram estabelecidos como critérios de inclusão: todas as categorias de artigos completos (original, revisão de literatura, reflexão, atualização, relato de experiência etc.); aqueles publicados em português entre os anos 2000 e 2017 e artigos que contivessem em seus títulos e/ou resumos os descritores em ciências da saúde (DeCS) "aconselhamento", "HIV” "AIDS". Os critérios de exclusão dos artigos foram: artigos repetidos nas bases de dados, artigos que não tenham o foco no aconselhamento em HIV/AIDS, que tratem sobre determinadas etapas da vida (Ciclo gravídico, puerperal, adolescência etc) e os estudos que não foram realizados no Brasil.

3. Categorização dos estudos:

Os descritores escolhidos para as buscas foram combinados em cada base de dados. Para sistematização da avaliação dos selecionados, as informações foram extraídas, utilizando-se adaptação de instrumento validado por Ursi (2006).

Os artigos foram classificados e os resultados encontrados foram posteriormente sintetizados, considerando a similaridade de conteúdo. 
4. Avaliação dos estudos incluídos na revisão:

Com base na categorização dos estudos, procedeu-se à avaliação das publicações com base nos objetivos, metodologia, resultados e conclusão destas, obtendo as contribuições de cada estudo para a síntese do conhecimento.

5. Discussão e interpretação dos resultados:

Nessa fase, procedeu-se à análise das publicações selecionadas, tendo como subsídio a questão norteadora do presente estudo. Buscou-se estabelecer os pontos de convergência e divergência entre os artigos, independente do tipo de pesquisa desenvolvida (quantitativa, qualitativa, quanti-qualitativa, artigos de revisão e relatos de experiência).

6. Síntese do conhecimento evidenciado nos artigos analisados:

Utilizou-se a subdivisão do conhecimento em duas etapas:

a) Atuação dos profissionais de enfermagem em aconselhamento no CTA: Relatando como deve ocorrer a práxis do enfermeiro diante da complexidade de um paciente com diagnóstico de HIV/AIDS;

b) O aconselhamento como cuidado de enfermagem aos indivíduos com HIV/AIDS.

Outra variável importante analisada foi o nível de evidência científica, esta também visa o fortalecimento do processo de PBE, dispondo de aparelhos de categorização de evidências caracterizados de modo hierárquico, a partir da abordagem metodológica seguida, a saber: Nível 1: evidências a partir da meta-análise de múltiplos estudos clínicos controlados e randomizados; Nível 2: evidências obtidas em estudos individuais com delineamento experimental; Nível 3: evidências de estudos quase-experimentais; Nível 4: evidências de estudos descritivos (nãoexperimentais) ou com abordagem qualitativa; Nível 5: evidências provenientes de relatos de caso ou de experiência; Nível 6: evidências baseadas em opiniões de especialistas (SOUZA, 2010; CARVALHO, 2009).

\section{Resultados e Discussão}

A busca pelos descritores HIV, AIDS, aconselhamento, enfermagem e cuidado, utilizando o booleano AND, nas bases de dados possibilitou encontrar 553 artigos, dos quais, após a aplicação dos critérios de inclusão e exclusão, obteve-se 9 artigos da base de dados LILACS e 8 da SCieLO, totalizando 17 artigos. 
O fluxograma elaborado segundo Moher (2009), a estratégia PRISMA, para a síntese dos dados coletados, onde a seguir estão dispostos a quantidade dos referidos trabalhos encontrados, selecionados e excluídos está disposto na figura 1 a seguir.

Figura 1. Fluxograma com os artigos encontrados, selecionados e excluídos da pesquisa, orientado pela estratégia PRISMA (MOHER, 2009. Pesqueira, PE, Brasil (2017).

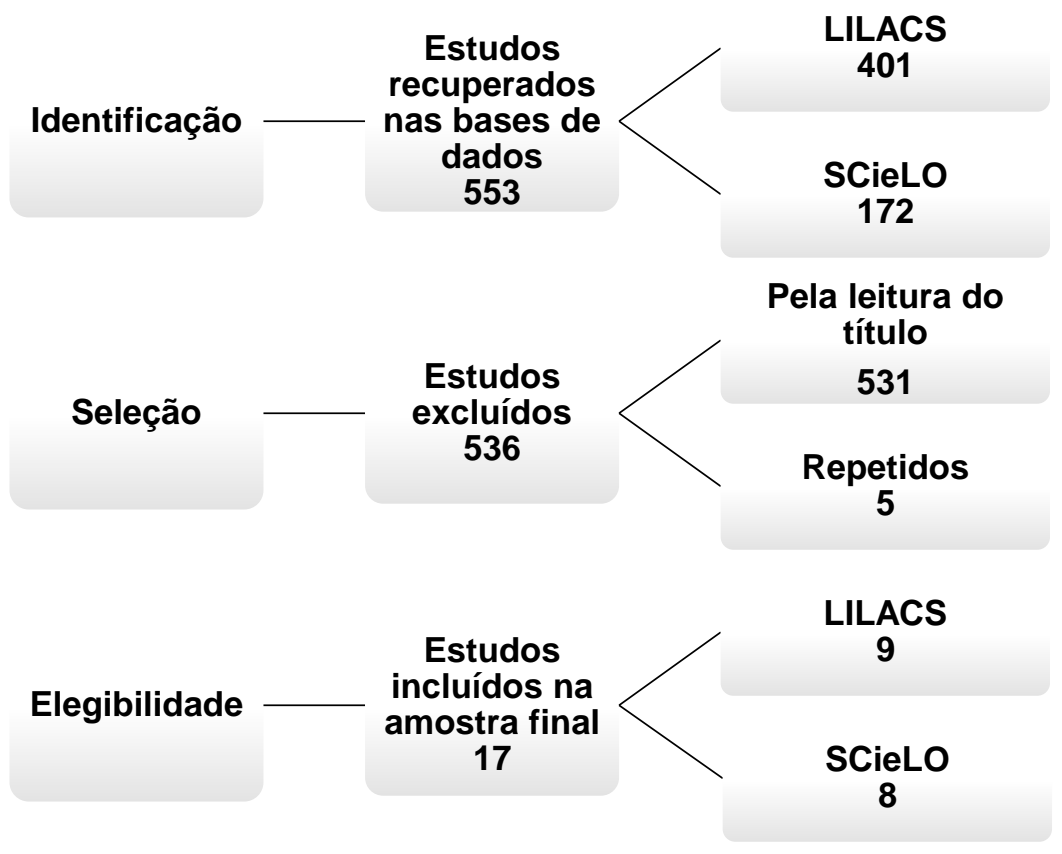

Os artigos da amostra final foram organizados em quadro sinótico e nas categorias título, autores, ano de publicação, objetos da pesquisa, periódico onde houve a publicação, estado onde a pesquisa ocorreu e delineamento da pesquisa / nível de evidência científica, com o intuito de sintetizar as informações de maneira breve, formando um banco de dados de fácil acesso e manejo. 
Quadro 1: Caracterização dos artigos selecionados sobre aconselhamento dos Enfermeiros em HIV/AIDS. Pesqueira, 2017.

\begin{tabular}{|c|c|c|c|c|c|c|c|c|}
\hline Númerc & Título & Autores & Ano & $\begin{array}{l}\text { Objeto de } \\
\text { estudo }\end{array}$ & Periódico & $\begin{array}{l}\text { Área do } \\
\text { Periódico }\end{array}$ & Estado & $\begin{array}{l}\text { Delineamento do } \\
\text { estudo / Nível de } \\
\text { evidência }\end{array}$ \\
\hline A1 & $\begin{array}{l}\text { Necessidades de } \\
\text { qualificação da } \\
\text { equipe de } \\
\text { enfermagem para a } \\
\text { assistência aos } \\
\text { clientes portadores do } \\
\text { HIV e da AIDS }\end{array}$ & $\begin{array}{l}\text { GRYSCHEK, L. } \\
\text { P. F. L. } \\
\text { A/DOMINGOS, } \\
\text { C. } \\
\text { J/FIGUEIREDO, } \\
\text { L. D. R. C }\end{array}$ & 2000 & $\begin{array}{l}\text { Questionários } \\
\text { com profissionais } \\
\text { de enfermagem }\end{array}$ & Rev. Esc. Enf. USP & Enfermagem & SP & N4 \\
\hline A2 & $\begin{array}{l}\text { Aconselhamento em } \\
\text { HIV/AIDS: Análise à } \\
\text { luz de Paulo Freire. }\end{array}$ & $\begin{array}{l}\text { MIRANDA, K. C. } \\
\text { L.;/ BARROSO, } \\
\text { M. G. T. }\end{array}$ & 2007 & $\begin{array}{l}\text { Profissionais do } \\
\text { aconselhamento } \\
\text { e Paulo Freire }\end{array}$ & $\begin{array}{l}\text { Rev Latino } \\
\text { Americana de } \\
\text { enfermagem }\end{array}$ & Enfermagem & $\mathrm{CE}$ & N4 \\
\hline A3 & $\begin{array}{c}\text { Considerações } \\
\text { sobre os discursos do } \\
\text { aconselhamento } \\
\text { nos centros de } \\
\text { testagem anti-HIV }\end{array}$ & $\begin{array}{l}\text { SOUZA, V.I } \\
\text { CZERESNIA }\end{array}$ & 2007 & $\begin{array}{l}\text { Contextualizada } \\
\text { por Fairclough. }\end{array}$ & $\begin{array}{c}\text { Interface - } \\
\text { Comunicação, } \\
\text { Saúde, Educação }\end{array}$ & Enfermagem & MG & $\mathrm{N} 4$ \\
\hline A4 & $\begin{array}{l}\text { Aconselhamento na } \\
\text { prevenção do HIV: } \\
\text { olhar dos usuários de } \\
\text { um centro de } \\
\text { testagem. }\end{array}$ & $\begin{array}{c}\text { SOUZA, V./ } \\
\text { CZERESNTA, D./ } \\
\text { NATIVIDADE, C. }\end{array}$ & 2008 & $\begin{array}{l}\text { Usuários no pós- } \\
\text { teste }\end{array}$ & $\begin{array}{c}\text { Caderno de Saúde } \\
\text { Pública }\end{array}$ & Enfermagem & MG & $\mathrm{N} 4$ \\
\hline A5 & $\begin{array}{l}\text { Reflexões sobre o } \\
\text { aconselhamento em } \\
\text { HIV/AIDS em uma } \\
\text { perspectiva freireana }\end{array}$ & $\begin{array}{l}\text { MIRANDA, K. C. } \\
\text { L/ BARROSO, M. } \\
\text { G. T./ SILVA, M. } \\
\text { R. F. }\end{array}$ & 2008 & $\begin{array}{l}\text { Perspectiva de } \\
\text { Paulo Freire }\end{array}$ & $\begin{array}{l}\text { Revista Brasileira } \\
\text { de Enfermagem }\end{array}$ & Enfermagem & $\mathrm{CE}$ & $\mathrm{N} 4$ \\
\hline
\end{tabular}


A6 Avaliação do autoestima de portadores de HIV/AIDS do município de Alfenas, Minas Gerais, Brasil. Auto-proteção rofissional e cuidado de enfermagem ao paciente soropositivo ao HIV: duas facetas de uma representação

A8 Subjetividade no GALINDO, W. C. 2013 aconselhamento em M./ FRANCISCO, HIV/AIDS.

A. L./

RIOS, L. F.

A9 A Instrução e a GALINDO, W. C. 2013 Relação como Modos

$$
\text { M./ }
$$

de Aconselhamen em HIV/Aids

CISCO, A

$$
\text { L./ }
$$

RIOS, L. F.

A10 Gestão e processos de HAAG, C.B./ trabalho nos centros GONÇALVES, T. de Testagem e

aconselhamento de BARCELLOS, N.

Porto Alegre-RS na perspectiva de seus aconselhadores

A11 Aconselhamento em PEQUENO, C. S./ 2013 HIV/AIDS:pressupo- MACÊDO, S. M./ tos teóricos MIRANDA, K. C.
Entrevista com enfermeiros e

técnicos com

auxílio de

questionário

Manuais do

Ministério da saúde

Aconselhadores do CTA

Temas em

Psicologia

Revista de Saúde Coletiva

Teorias de psicologia
Descritivo, exploratório, de delineamento transversal 
para uma prática

clínica fundamentada

A12 Proposições para a

formação de

GALINDO, W. C. 2013 aconselhadores em A. L./ HIV/AIDS.

RIOS, L.F.

A13 Reflexões sobre o GALINDO, W. C. 2015 trabalho de

$$
\text { M./ }
$$

aconselhamento em FRANCISCO, A. HIV/AIDS,

$$
\text { L./ }
$$

RIOS, L. F.

A14 Aconselhamento em BARBOSA, A. L 2015 doenças sexualmente T/ GOMES, X. M. transmissíveis na L/ HOLZMANN, atenção primária: F. P. A/ PAULA, percepção e prática

B. M. A/ profissional HAIKAL, A. S. D

A15 Cuidado de MACÊDO, M. enfermagem em S/MIRANDA, L.

Serviço Ambulatorial C. K/SILVEIRA, Especializado em C. L/GOMES,T. HIV/AIDS M. A

A16

$$
\text { Valores }
$$

DANTAS, C. 2015 intervenientes no

F/DANTAS, C. C cuidado de enfermagem ao cliente HIV/AIDS

A17 Potencialidades e fragilidades da rede

MEIRELLES, S. 2015 de cuidado da pessoa H. B/TONNERA. J. C. L com HIV/AIDS

Manuais do

Ministério da saúde e

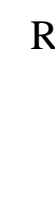

conselhadores

Manuais do

Ministério da saúde
Revista de Saúde Psicologia
Coletiva

PE

Temas em

Psicologia

PE

N4

Psicologia

Acta Paul Enferm. Enfermagem

MG

N4

Questionários

com enfermeiros

e médicos

2015

Entrevista aberta

Revista Brasileira

Enfermagem

RJ

direta com diário

de campo

Entrevista

Revista Aquichan

RJ

sistema digital

Entrevista com Revista Brasileira

de Enfermagem

SC

N4 
Posteriormente, foram extraídos os conceitos abordados em cada artigo e de interesse das pesquisadoras. Os trabalhos foram comparados e agrupados por similaridade de conteúdo, sob a forma de categorias empíricas, sendo construídas duas categorias para análise, assim especificadas: Atuação dos profissionais de saúde e enfermeiros em aconselhamento no CTA e O aconselhamento como cuidado de enfermagem aos indivíduos com HIV/AIDS.

\section{Atuação dos profissionais de saúde e enfermeiros em aconselhamento no CTA}

O aconselhamento é um dispositivo de saúde ancorado em três componentes fundamentais - o apoio emocional, o apoio educativo e o apoio de risco - devendo ser realizado por um profissional de saúde capacitado. Através das descrições dos tipos de aconselhamento em HIV/aids utilizados nos CTAs e das sugestões para a melhora do aconselhamento nestes centros, foi possível analisar que a forma como o aconselhamento é realizado pode interferir de maneira positiva ou negativa na vida de diversos usuários que usufruem desse serviço (SOUZA et. al., 2008; SOUZA, CZERESNIAS, 2007; BRASIL, 2004; 2000).

Há diferentes práticas nos tipos de aconselhamentos utilizados nos CTAs pelos profissionais de saúde que estariam prejudicando a adesão dos usuários a realização da sorologia anti-HIV e a procura pelo serviço. Nesse sentido observa-se no aconselhamentoinstrução um caráter informativo, centralizado no saber do profissional de saúde, tendo o usuário responsável por sua condição sorológica, inclusive pela redução dos índices de infecção pelo HIV (GALINDO, FRANCISCO, RIOS, 2015). Corroborando com essa prática, considerase que os profissionais de saúde concentram o aconselhamento em um roteiro pré-elaborados que não possibilitam o diálogo com os usuários (BRASIL, 2004). Para Galdino (2015), de forma tênue, observa-se a prática do aconselhamento-relação, prática construída entre profissional de saúde e usuário, que valoriza a singularidade do sujeito, respeitando sua compreensão a respeito dos temas abordados.

No que se refere ao aconselhamento pré-teste (aquele realizado antes do exame de sangue) sendo considerado de suma relevância por seu caráter informativo e pós-teste (realizado na entrega do resultado) tido como esclarecedor e tranquilizador ao usuário, estes procedimentos respectivamente trabalham de modo individual ou coletivo, tendo no pós-teste a entrega do resultado e um aconselhamento individual com duração de 20 à 30 minutos, já no

1203 Id on Line Rev. Mult. Psic. V.12, N. 40. 2018 - ISSN 1981-1179 Edição eletrônica em http://idonline.emnuvens.com.br/id 
aconselhamento pré-teste coletivo, este acontece de maneira específica e distinta tendo uma duração de 60 à 90 minutos, estes dois aconselhamentos são discutidos (SOUZA et. al., 2008; SOUZA, CZERESNIAS, 2007; MIRANDA et. al., 2008) •

Ao analisar os artigos o que concerne a prática do aconselhamento, aponta-se para uma (re) construção do "saber fazer" do outro com vista na sua autonomia em relação à prevenção. No entanto, o cotidiano dos serviços, observações, concepções simplistas e reducionistas em relação ao trabalhador saúde-usuário, com dificuldades de diálogos com as pessoas vivendo com HIV/Aids. Faz-se necessário que o aconselhador utilize em sua prática, algumas bases de algum princípio teórico em relação ao aconselhamento, tornando seu saber/fazer fundamentado (SOUZA, CZERESNIAS, 2010; SOUZA et. al., 2008).

Quanto à atuação dos profissionais este devem utilizar metodologias participativas, para romper a idéia que o aconselhamento é uma "tecnologia dura" mecanizada, com rígidas orientações. Deve-se investir na formação deste aconselhador com uma visão compreensão/estudo das diversas implicações das dimensões que compõe sua prática de atuação (SOUZA et. al., 2008).

Vale ressaltar que os artigos A11, A12, A13, A14, A15, A16 e A17, não contribuíram significativamente para esta vertente pesquisada, porém dão embasamento teórico/metodológico para a assistência de enfermagem ao indivíduo com HIV/AIDS.

\section{O aconselhamento como cuidado de enfermagem aos indivíduos com HIV/AIDS}

Esta categoria retrata que o modelo de cuidar ao portador de HIV/Aids está pautado em três pilares: no profissional, na patologia e no usuário. Os profissionais devem reconhecer os limites de seus valores e não permitir que estes conduzam para um cuidado discriminatório (MENDES, 2012; FRANÇA, 2000).

Corroborar com essas reflexões Souza et. al., (2008) e Souza e Czeresnias (2007) no que concerne o aconselhamento em HIV/aids como prática da enfermagem consiste em uma estratégia que auxiliar o indivíduo assistido na diminuição dos estigmas e a vulnerabilidade, nas práticas preventivas. Para Fraça (2000), os profissionais devem se livrar dos seus próprios preconceitos e auxiliar o outro de maneira holística independentemente da sua patologia. Nessa linha de raciocínio em se tratando de IST/aids lida-se com o cotidiano das relações humanas, 
às vezes conflituosas e nem sempre explícitas, que podem dificultar as ações preventivas na assistência prestada (MOREIRA, 2015).

Neste contexto, os profissionais enfrentam dificuldades em lidar com aspectos subjetivos e íntimos, envolver a sexualidade e conjugalidade no aconselhamento aos usuários. Há uma necessidade de melhorar sua prática de aconselhamento baseado na ética e na integralidade. Os indivíduos precisam ter a definição do papel que desempenham em seu processo de trabalho, determinar seu lugar na sociedade e a posição que faz parte de suas expectativas (LINS, 2000). O aconselhamento deve está inserido nos serviços de saúde enquanto prática para possibilitar uma interação entre trabalhador da área da saúde e o usuário, proporcionar mútua troca de conhecimentos e sentimentos, abrindo caminho para a superação de conflitos (BRASIL, 2008).

Vale ressaltar que para os profissionais prestarem um aconselhamento de qualidade, é necessário que estes estejam qualificados para desempenhar um bom serviço e para ajudar esses indivíduos, com competências e habilidades teóricas e práticas, atitudes, hábitos e valores éticos, para a realização de um trabalho eficaz (SOUZA, CZERESNIAS, 2010; MOREIRA,2015; BARBOSA et al. 2015;BRITO et al., 2009; FORMOSO; OLIVEIRA,2009;GALINDO; FRANCISCO; RIOS, 2013).

Um grande desafio para enfermagem é a possibilidade de refletirem sobre a sua prática e seu poder enquanto sujeitos capazes de realizar mudanças que impliquem em uma melhor qualidade de assistência (MOREIRA, 2015).

Enfim, aconselhamento é um paradoxo que gera conforto e conflitos, simultaneamente (HAAG; GONÇALVES E BARCELLOS， 2013; MOREIRA， 2015; GALINDO; FRANCISCO; RIOS, 2013). Entretanto, é uma estratégia crucial, na quebra da cadeia de transmissão. O ideal, é que em um momento em que o profissional precisa utilizar-se de teorias para fundamentar a sua prática utilize-se de metodologias participativas, como por exemplo, a perspectiva educativa de Paulo Freire, a teoria existencial ou a teoria comportamental, por exemplo. Tornando-a uma práxis que aproxime o profissional do usuário, promovendo uma melhor assistência e assim, melhores resultados (GALINDO; FRANCISCO; RIOS, 2015; MIRANDA et al., 2008; FORMOSO; OLIVEIRA,2009).

\section{CONCLUSÃO}

O modelo biomédico ainda está impregnado na maioria das ações de aconselhamento, pois de acordo com as literaturas analisadas, os serviços prestados são mais tradicionais, 
verticais, instrutivos e centrados no aconselhador, que de um modo geral, impõe suas noções ideologias como as únicas a serem consideradas. Podendo afastar o usuário, por não se sentir realmente acolhidos.

O aconselhamento é uma estratégia de relevância que pode ser utilizada na prevenção de infecções/doenças e seus agravos, principalmente aquelas evitáveis como o HIV/aids, a qual se faz necessário que aconteça cada vez mais a orientação, mesmo diante das fragilidades e potencialidades do serviço. Os CTAs são um serviço importante de diagnóstico e prevenção em HIV/Aids e têm sido reconhecidos em oferecerem, confiabilidade no resultado dos exames, pela garantia de sigilo, ética e respeito.

Nesse sentido, o aconselhamento ainda não se configura como um momento educativo, por não ser dialogal, não estimular a consciência crítica para a intervenção da realidade e autonomia do sujeito. No entanto, o aconselhamento para produzir uma resposta positiva, é necessário uma maior aproximação do usuário com o aconselhador, deixando-o mais confiante para relatar sua história, medos e dificuldade, pois de acordo com a complexidade dessa prática, o grande desafio para os profissionais em formação para o exercício do aconselhamento é o de fortalecimento no processo formativo, a partir do uso efetivo de metodologias participativas, assim é necessário que o profissional esteja capacitado para analisar o individuo como um todo, levando em consideração que o mesmo possui características biopsicossociais distintas e que precisam ser respeitadas.

\section{Referências}

BARBOSA, A. et al. Aconselhamento em doenças sexualmente transmissíveis na atenção primária: percepção e prática profissional. Acta Paulista de enfermagem. v. 28, n. 6. 2015.

BRASIL. Ministério da Saúde (MS). Secretaria de Vigilância em Saúde. Programa Nacional de DST e Aids. Boletim Epidemiológico - AIDS e DST. Brasília, 2011.

BRASIL. Ministério da Saúde (MS). Secretaria de Vigilância em Saúde. Programa Nacional de DST e Aids. Centros de Testagem e Aconselhamento do Brasil - Desafios para a equidade e o acesso. Brasília, 2008.

BRASIL. Senado Federal. Constituição da República Federativa do Brasil. Brasília, 1988.

BRASIL. Ministério da Saúde. Departamento de DST, Aids e Hepatites Virais. Diretrizes para organização e funcionamento dos CTA do Brasil. Brasília, 2010. 
BRASIL. Ministério da Saúde (MS). Secretaria de Vigilância em Saúde. Coordenação Nacional de DST/AIDS. Recomendações para a profilaxia da transmissão vertical do HIV e terapia antiretroviral em gestantes. Brasília, 2004.

BRASIL. Ministério da Saúde (MS). Coordenação Nacional de DST e AIDS. Aconselhamento em DST, HIV e AIDS: diretrizes e procedimentos básicos. Brasília, 2000.

BRITO, T. P. et al. Avaliação da auto-estima em portadores de HIV/Aids do município de Alfenas. Revista Gaúcha de Enfermagem, Minas Gerais, v. 30, n. 2, p. 190. 2009.

CARVALHO, D. et al. Teoria fundamentada nos dados-aspectos conceituais e operacionais: metodologia possível de ser aplicada na pesquisa em enfermagem. Revista latino-americana de Enfermagem, v.17, n. 4, 2009.

FORMOZO, G; OLIVEIRA, D. C. Auto-proteção profissional e cuidado de enfermagem ao paciente soropositivo ao HIV: duas facetas de uma representação. Acta Paulista Enfermagem, v. 22, n.4, p. 292$8,2009$.

GALINDO, W. C. M.; FRANCISCO, A. L.; RIOS, L. F.. A instrução e a relação como modos de aconselhamento em HIV/Aids. Temas em Psicologia, v. 21, n. 3, p. 989-1004, 2013.

GALINDO, W. C. M.; FRANCISCO, A. L.; RIOS, L. F. Subjetividade no aconselhamento em HIV/Aids. Revista Psicologia Política, v. 13, n. 26, p. 129-145, 2013.

GALINDO, W. C. M.; FRANCISCO, N. L.; RIOS, L. F. Reflexões sobre o trabalho de aconselhamento em HIV/AIDS. Temas em Psicologia, v. 23, n. 4, p. 815-829, 2015.

GARCIA, S; SOUZ, F. M. Vulnerabilidades ao HIV/aids no contexto Brasileiro: Iniquidades de gênero, raça e geração. Saúde Social. São Paulo, v. 19, p. 9-20, 2010.

GOMES, A. M. T. et al. As facetas do convívio com HIV: formas e relações sociais e representações sociais da aids para pessoas soropositivas hospitalizadas. Escola Anna Nery, v.16, n. 1. p. 111-120, 2012.

GRANEIRO, A. et al. Voluntary counseling and testing (VCT) services and their contribution to access to HIV diagnosis in Brazil. Caderno de Saúde Pública, 2009.

HAAG, C. B.; GONÇALVES, T. R.; BARCELLOS, N. T. Gestão e processos dec trabalho nos Centros de Testagem e Aconselhamento de Porto Alegre-RS na perspectiva dec seus aconselhadores. Physis: Revista de Saúde Coletiva, v. 23, n. 3, 2013.

HIRDES, A. KANTORSKI, L. P. Sistematização do cuidado em enfermagem psiquiátrica. Texto contexto em enfermagem, Florianópolis, v.9, n.2, p.104-105. 2000.

LINS, A. L. F. P et al. Necessidades de qualificação da equipe de enfermagem para a assistência aos clientes portadores do HIV e da AIDS. Revista da Escola de Enfermagem da USP, v. 34, n. 3, p. 288293, 2000.

MENDES, K. D. S.; SILVEIRA, R. C. C. P.; GALVÃO, C. M. Revisão integrativa: método de pesquisa para a incorporação de evidências na saúde e na enfermagem. Texto \& Contexto Enfermagem, v.17, n.4, p.58-64, 2008. 
MENDES, E.V. O cuidado das condições cronicas na atenção primária à saúde: o imperativo da consolidação da estratégia da saúde da família. 2012. 132f. Dissertação (Pós -Graduação em Enfermagem) - Organização Pan Americana da Saúde, 2012.

MIRANDA, K. C. L. et al. Reflexões sobre o aconselhamento em HIV/AIDS em uma perspectiva freireana. Revista brasileira de enfermagem, v. 61, n. 6, p. 899-903, 2008.

MOHER, D. et al. Preferred Reporting Items for Systematic Reviews and Meta-Analyses: The PRISMA Statement, 2009. Acesso em: 23 de julho de 2017. Disponível em: http://prismastatement.org/PRISMAStatement/FlowDiagram.aspx.

MOREIRA, W. C et al. Ações educativas do enfermeiro na promoção da saúde sexual e reprodutiva do adolescente. Revista Interdisciplinar, v. 8, n. 3, p. 213-220, 2015.

PUPO. L, R. Aconselhamento em DST/Aids: uma análise crítica de sua origem histórica e conceitual e de sua fundamentação teórica. 2007. Dissertação - Faculdade de Medicina da Universidade de São Paulo, São Paulo.

SILVA, L. M. S. et al. Integralidade em saúde: avaliando a articulação e a coresponsabilidade entre o Programa Saúde da Família e um serviço de referência em HIV/aids. Epidemiologia e Serviço de Saúde, Brasília, v.14, n.2, p. 97-104, 2005.

SOUZA, V.; CZERESNIA, D. Considerações sobre os discursos do aconselhamento nos centros de testagem anti-HIV. Interface-Comunicação, Saúde, Educação, v. 11, n. 23, p. 531-548, 2007.

SOUZA, V.; CZERESNIA, D.; NATIVIDADE, C. Aconselhamento na prevenção do HIV: olhar dos usuários de um centro de testagem. Caderno de saúde pública, v. 24, n. 7, p. 1536-1544, 2008.

TONNERA, L. J; MEIRELLES, B. S. Potencialidades e fragilidades da rede de cuidado da pessoa com HIV/AIDS. Revista Brasileira de Enfermagem, p. 438-444, 2015

UNAIDS. Tratamento 2015. ONUSIDA, 2015. Acesso em: 10 de outubro de 2017. Disponível em: http://unaids.org.br/wp-content/uploads/2016/03/Tratamento-2015.pdf.

\section{Como citar este artigo (Formato ABNT):}

VERAS, Samara Maria de Jesus; SANTOS, Kerllane R. F. do N.; LEITE-SALGUEIRO, Claudia Daniele B.; SANTOS, Silvana C. do; GONÇALVES, Cláudia Fabiane G.; ALEXANDRE, Ana Carla S.; CARVALHO, Valdirene Pereira da S. Reflexões sobre Cuidado e Aconselhamento de Profissionais de Saúde e Enfermeiros Em HIV/AIDS nos Centros de Testagem e Aconselhamento: Revisão Integrativa. Id on Line Revista Multidisciplinar e de Psicologia, 2018, vol.12, n.40, p.1193-1208. ISSN: 1981-1179.

Recebido: 28.05.2018

Aceito: 30.05 .2018 